\title{
A Biomedical Surface Enhanced Raman Scattering Substrate: Functionalized Three-Dimensional Porous Membrane Decorated with Silver Nanoparticles
}

\author{
Li Yuan, Jinghuai Fang, Yonglong Jin, Chaonan Wang, and Tian Xu \\ School of Science, Nantong University, No. 9 Se Yuan Road, Nantong, Jiangsu 226019, China \\ Correspondence should be addressed to Tian Xu; xutian@ntu.edu.cn
}

Received 4 September 2015; Accepted 10 November 2015

Academic Editor: Christoph Krafft

Copyright @ $2015 \mathrm{Li}$ Yuan et al. This is an open access article distributed under the Creative Commons Attribution License, which permits unrestricted use, distribution, and reproduction in any medium, provided the original work is properly cited.

\begin{abstract}
We fabricated a simple, cheap, and functional surface enhanced Raman scattering substrate for biomedical application. Hot spots between two close silver nanoparticles distributed in the skeleton of a three-dimensional porous membrane, especially in the pores, were formed. The dual poles of micropores in the membrane were discussed. The pores could protect the silver nanoparticles in the pores from being oxidized, which makes the membrane effective for a longer period of time. In addition, Staphylococcus aureus cells could be trapped by the micropores and then the Raman signal became stronger, indicating that the functional surface enhanced Raman scattering substrate is reliable.
\end{abstract}

\section{Introduction}

Surface enhanced Raman scattering (SERS) is widely used in biomedical sensing, chemical analysis, and environmental monitoring owing to its extremely high sensitivity. It is a powerful analytic tool in the detection of small organic molecule, such as deoxyribonucleic acid [1-3], proteins [4], tumor cells [5], bacteria [6,7], and enzymes [8]. The electromagnetic resonance properties of a substrate, such as a roughened surface on gold nanoparticles and silver nanoparticles, are used for generating the SERS effect. However, achieving a reliable, stable, and uniform SERS signal substrate is challenging.

To fabricate silver nanoparticle arrays with high SERS activity and better uniformity, several groups have prepared periodic nanostructure arrays via lithography [9-12]. The electric field of incident light is usually enhanced in the interstitial regions or the gaps among the closely spaced nanostructures, which are known as "hot spots." Therefore, the improvement of the intensity and density of hot spots is important for designing and fabricating SERS substrates. The preparation of hot spots on a two- or three-dimensional surface is a good method for increasing the density of hot spots. Wang et al. have fabricated hierarchical silver nanocap-type array using a porous anodic aluminum oxide template, in which hot spots are engineered on a threedimensional surface [13]. In addition, extremely high SERS activity was observed on the three-dimensional surface with the hot spots. Jung et al. have developed a chitosan-silver nanoparticle hybrid three-dimensional porous structure as a SERS substrate for biomedical applications [1]. However, the function of the pores in the membrane was not analyzed.

Here we attempted to develop a simple and functional surface enhanced Raman scattering substrate with hot spots distributed in the skeleton of a three-dimensional porous membrane, especially in the pores. Its potential use for biomedical application was evaluated.

\section{Method}

2.1. Preparation of Surface Enhanced Raman Scattering Substrate. Silver nitrate, glucose, and ammonium hydroxide were purchased from China Sinopharm Chemical Reagent Company Limited, and the filter membrane was purchased from Haining Zhenghao Filter Company Limited. The experimental steps are as follows: (1) cleaning the filter membrane: the membrane was soaked in $70^{\circ} \mathrm{C}$ deionized water for 2 


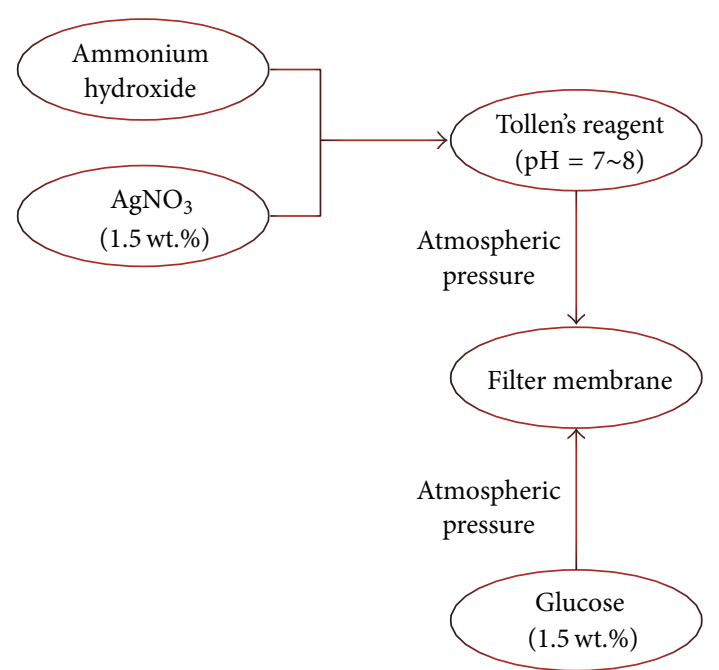

FIGURE 1: Schematic representation of the experiment.

hours after being cleaned 5 times and then dried in vacuo. (2) Preparation of Tollen's reagent: ammonium hydroxide was added to $20 \mathrm{~mL} \mathrm{AgNO}_{3}$ (1.5 wt\%) until the mixed solution became transparent and $\mathrm{pH}=7-8$. The corresponding reaction equations are

$$
\begin{aligned}
2 \mathrm{Ag}^{+}+2 \mathrm{OH}^{-} & \longrightarrow \mathrm{Ag}_{2} \mathrm{O} \downarrow+\mathrm{H}_{2} \mathrm{O} \\
\mathrm{Ag}_{2} \mathrm{O}+4 \mathrm{NH}_{3}+\mathrm{H}_{2} \mathrm{O} & \longrightarrow 2\left[\mathrm{Ag}\left(\mathrm{NH}_{3}\right)_{2}\right]^{+}+2 \mathrm{OH}^{-} \\
\mathrm{NH}_{4}^{+}+\mathrm{OH}^{-} & \longrightarrow \mathrm{NH}_{3} \cdot \mathrm{H}_{2} \mathrm{O}
\end{aligned}
$$

A membrane was placed on the filter. As shown in Figure $1,1.0 \mathrm{~mL}$ of Tollen's reagent was added from the top of the filter, and $1.0 \mathrm{~mL}$ glucose $(1.5 \mathrm{wt} \%)$ was added to the opposite side. For adequate reaction of the two solutions, atmospheric pressure (approximately $0.6 \mathrm{~N} / \mathrm{cm}^{2}$ ) was simultaneously introduced to the top and bottom of the membrane in the filter. Subsequently, the filter membrane was immersed in a $65^{\circ} \mathrm{C}$ water bath for $1 \mathrm{~min}$. Finally, the membrane was washed with deionized water and dried in a vacuum oven at room temperature.

The reduction equation is

$$
\begin{aligned}
& \mathrm{C}_{6} \mathrm{H}_{12} \mathrm{O}_{6}+2 \mathrm{Ag}\left(\mathrm{NH}_{3}\right)_{2} \mathrm{OH} \\
& \quad \longrightarrow \mathrm{C}_{5} \mathrm{H}_{11} \mathrm{O}_{5} \mathrm{COONH}_{4}+2 \mathrm{Ag} \downarrow+3 \mathrm{NH}_{3}+\mathrm{H}_{2} \mathrm{O}
\end{aligned}
$$

2.2. Morphology of Surface Enhanced Raman Scattering Substrate and the Time of Its Effectiveness. Scanning electron microscopy (SEM, Hitachi S3400) was used to investigate the porous membrane decorated with silver nanoparticles. The SEM images were recorded in a $15 \mathrm{kV}$ accelerating voltage. Then, we investigated the effective time of the decorated porous membrane using crystal violet $(\mathrm{CV}$, from China Sinopharm Group Company Limited) as Raman probes. For comparison, $1.0 \mathrm{~mL}$ of Tollen's reagent and $1.0 \mathrm{~mL}$ of glucose (1.5 wt\%) were added on a glass slide (without pores), which was heated to $65^{\circ} \mathrm{C}$. Then, the glass substrate and porous

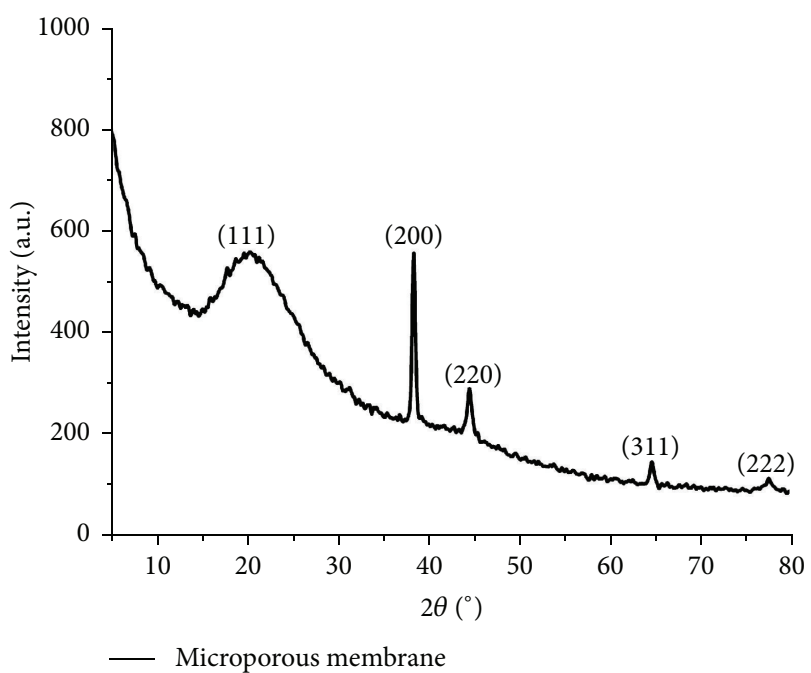

FIGURE 2: XRD pattern of microporous membrane decorated with silver nanoparticles.

membrane with the silver nanoparticles were exposed to air. Every $24 \mathrm{~h}, \mathrm{CV}\left(1 \times 10^{-5} \mathrm{M}\right)$ was deposited on the two substrates (the glass substrate and porous membrane with silver nanoparticles), and the corresponding Raman signals were collected.

2.3. Surface Enhanced Raman Scattering Measurement. After the sample $(25 \mu \mathrm{LCV})$ was deposited on the silver nanoparticles porous membrane, the membrane was dried in a vacuum oven at room temperature. For the biomedical application, the bacteria (S. aureus ATCC 27217, purchased from China Center of Industrial Culture Collection) were trapped in the pores of membrane to increase the contact area with the silver nanoparticles. First, a solution of S. aureus was cleaned five times with deionized water, and the porous membrane decorated with silver nanoparticles was divided into five pieces of equal size. Then, the parameter $B_{\text {in }}$, which is the ratio of $S$. aureus trapped inside the micropores, was introduced. Subsequently, the five pieces of the silver nanoparticles porous membrane were immersed in the solution of $S$. aureus with turbidity $(2.7 \mathrm{MCF})$, and then, the mixtures were centrifuged at different rotation speeds $(12000,10000$, 8000,6000 , and $4000 \mathrm{rmp}$ ) for $3 \mathrm{~min}$. Different values of $B_{\text {in }}$ were obtained due to the different rotation speeds. After being dried in a vacuum oven at room temperature, the membranes were examined by an Advantage Near Infrared Raman Spectrometer provided by DeltaNu Corporation. Here, the laser source for excitation was a $785 \mathrm{~nm}$ diode laser with $100 \mathrm{~mW}$ of power. The integration time was $5 \mathrm{~s}$, and a resolution of $8 \mathrm{~cm}^{-1}$ was used. NuSpec software was used to analyze the spectra.

\section{Results}

3.1. Morphology of the SERS Substrate. Figure 2 shows the Xray diffraction pattern of microporous membrane decorated with silver nanoparticles. It is shown that there are some 


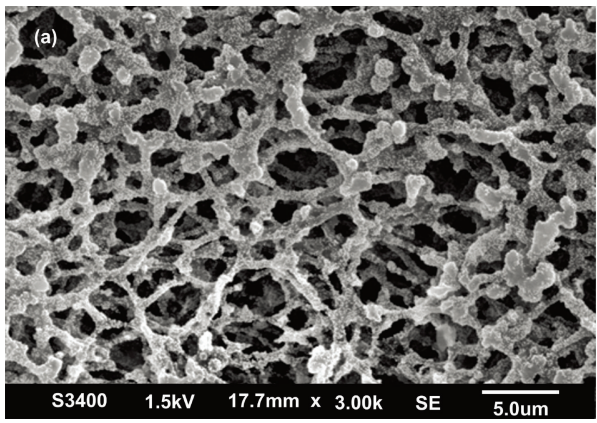

(a)

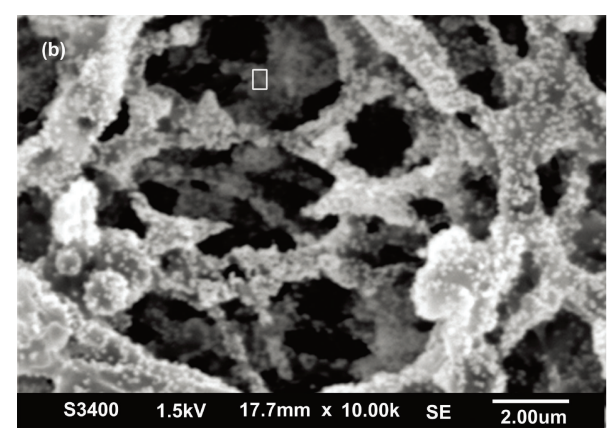

(b)

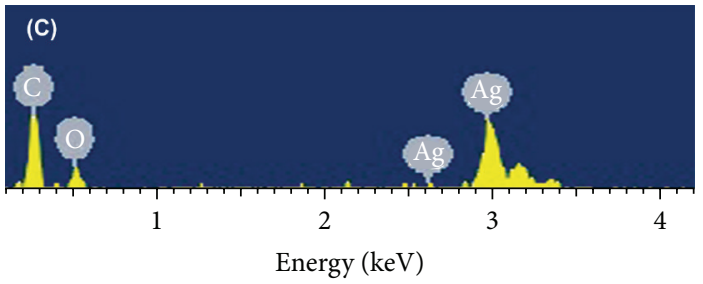

(c)

FIGURE 3: Representative scanning electron microscopy images of the membrane: (a) silver nanoparticles reduced membrane; (b) magnification of (a); (c) X-ray energy dispersive spectroscopy of the rectangle in (b).

amounts of silver nanoparticles here and there in the membrane.

The morphology of the SERS substrate was observed using SEM, as shown in Figure 3. Figures 3(a)-3(b), which show the SEM images of the decorated porous membrane under different magnifications, indicate that the silver nanoparticles have been immobilized in an isolated state in the membrane. X-ray energy dispersive spectroscopy (EDX) results (Figure 3(c)) indicate that the silver nanoparticles were uniformly distributed inside the pores.

Based on the porous structure, the silver nanoparticles were dispersed in the skeleton of the membrane and in the pores. The gaps between closely spaced silver nanoparticles, which are known as "hot spots," were densely dispersed on the membrane.

It is well known that the SERS effect of silver nanoparticles is outstanding without considering their stability. Therefore, it is important to prolong the effective usage time of silver nanoparticles. The micropores in the membrane allow for the protection of the silver nanoparticles from direct exposure to the air. The Raman spectra of CV $\left(1 \times 10^{-5} \mathrm{M}\right)$ in the two substrates (i.e., silver nanoparticles on glass and silver nanoparticles in a porous membrane) were investigated daily. The Raman shift at $1165 \mathrm{~cm}^{-1}$ was chosen for the characteristic peaks of crystal violet. The Raman signal of it is shown in Table 1. For the silver-coated glass substrate, the Raman signal at $1165 \mathrm{~cm}^{-1}$ decreased to less than 500 after 10 days. However, this peak was still observed after 15 days for the silver-coated porous membrane, supporting that the effective usage time of the decorated porous membrane is longer than that of glass substrate. The comparison of the Raman spectra for the glass substrate and membrane on the fifth day is shown
TABLE 1: The comparison of two substrates (microporous membrane and glass) for Raman signal at $1165 \mathrm{~cm}^{-1}$.

\begin{tabular}{lcc}
\hline Time (day) & Microporous membrane & Glass \\
\hline 1 & 5769 & 5760 \\
2 & 5287 & 5211 \\
3 & 5051 & 4664 \\
4 & 4945 & 4558 \\
5 & 4439 & 4027 \\
6 & 4108 & 3982 \\
7 & 3805 & 3343 \\
8 & 3609 & 2484 \\
9 & 3120 & 1280 \\
10 & 2800 & 523 \\
11 & 2510 & $<500$ \\
12 & 2325 & $<500$ \\
13 & 2008 & $<500$ \\
14 & 1385 & $<500$ \\
15 & 920 & $<500$ \\
\hline
\end{tabular}

Microporous membrane is the porous substrate coated with Ag nanoparticles.

Glass is the glass substrate coated with Ag nanoparticles.

in Figure 4. Comparing with the glass substrate for Raman signal at $1165 \mathrm{~cm}^{-1}$, the percentage coefficient of variation for the spectra is about $10.2 \%$.

The silver nanoparticles on glass substrate were more easily oxidized compared to those in the porous membrane. The pores of the membrane increased the specific surface areas of the substrate and simultaneously prevent AgNP from 


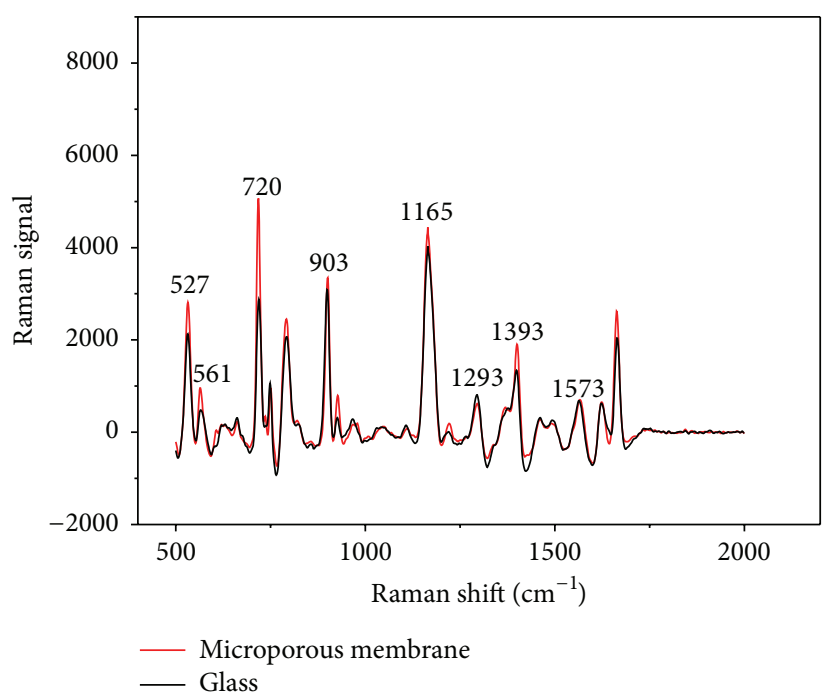

FIGURE 4: Surface enhanced Raman scattering spectra of $25 \mu \mathrm{L}$ crystal violet molecules $\left(1 \times 10^{-5} \mathrm{M}\right)$ on the silver nanoparticles coated porous substrate and glass substrate on the fifth day.

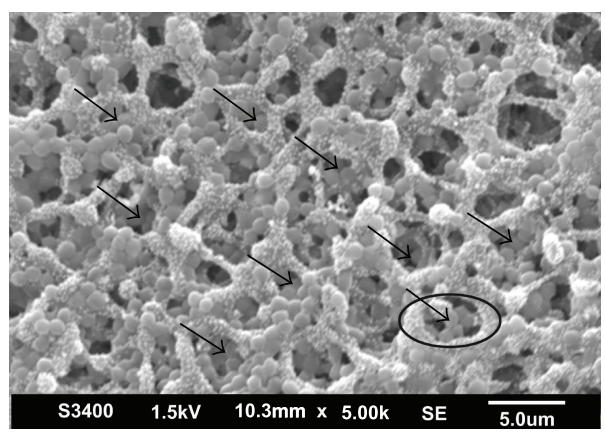

(a)

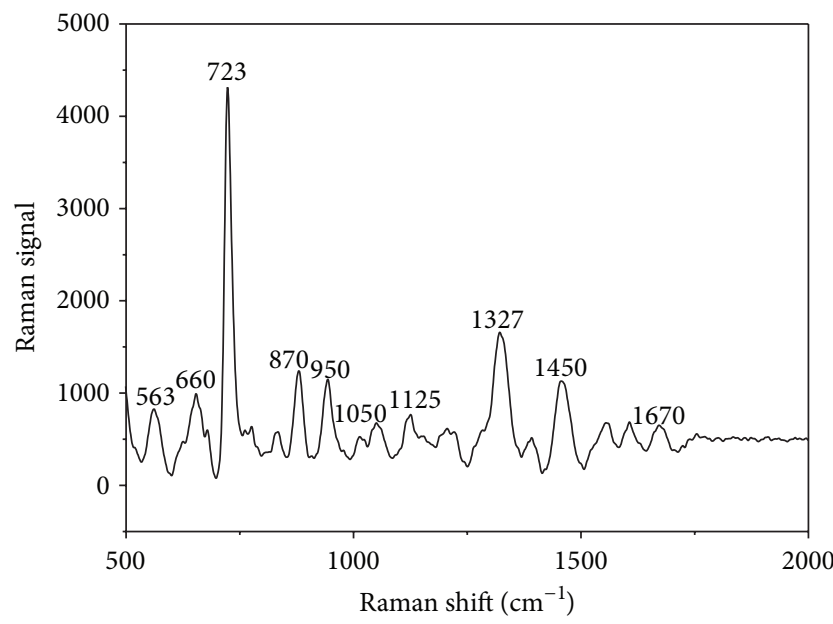

(b)

Figure 5: (a) Representative scanning electron microscopy image of Staphylococcus aureus trapped in the porous silver nanoparticles membrane; (b) surface enhanced Raman scattering spectra of Staphylococcus aureus when $B_{\text {in }}=0.683$ ( $B_{\text {in }}$ is the ratio of Staphylococcus aureus trapped inside the micropores).

being oxidized, which is the main reason for enhancing the Raman signal of the porous membrane.

3.2. Biomedical Applications. Another important effect of the pores was investigated. We determined the effect of the percentage of $S$. aureus in the micropores $B_{\text {in }}$ on the Raman intensity. Herein, each value of $B_{\text {in }}$ was obtained by an average of nearly 20 SEM images (approximately 971 bacteria in each piece of the silver nanoparticles membrane), and the results are shown in Figure 5 and Table 2.

When $B_{\text {in }}=0.683$, many bacteria were imprisoned in the pores (Figure 5(a)). The corresponding SERS spectrum is shown in Figure 5(b).

The Raman peak at $723 \mathrm{~cm}^{-1}$, which corresponds to the ring breathing of the adenine ring, was chosen as
TABLE 2: Raman signal of Staphylococcus aureus at $723 \mathrm{~cm}^{-1}$ in different values of $B_{\text {in }}$ ( $B_{\text {in }}$ is the ratio of Staphylococcus aureus trapped inside the micropores).

\begin{tabular}{lc}
\hline$B_{\text {in }}$ & Raman signal \\
\hline 0.683 & 4315 \\
0.664 & 2464 \\
0.642 & 1951 \\
0.623 & 1763 \\
0.605 & 1558 \\
\hline
\end{tabular}

the characteristic peak for $S$. aureus. For different values of $B_{\text {in }}$, the Raman signal at $723 \mathrm{~cm}^{-1}$ was investigated in Table 2 . The decorated microporous membrane can hold enough 
S. aureus cells to enhance the interaction between the bacteria and the silver nanoparticles or hot spots. Therefore, the Raman signal at $723 \mathrm{~cm}^{-1}$ increases as the number of bacteria trapped in the pores increases.

\section{Discussion}

SERS is an abnormal surface optical phenomenon resulting in strongly increased Raman signals for molecules adsorbed on nanostructured coinage metals. SERS has thus been an object of great interest in many areas of including chemical analysis, corrosion, lubrication, heterogeneous catalysis, biological sensors, and molecular electronics [3-6]. However, it is still a challenge in controlling the size and morphology of nanoparticles and their aggregates, the packing degree of assemblies, and the interparticle gap $[10,12]$. Therefore, the fabrication of a reliable, stable, and uniform SERS signal substrate remains demanded until now.

In this paper, we present a rapid and simple method for the fabrication of a silver porous membrane, in which the silver nanoparticles are distributed in the membrane, especially in micropores. The nanoparticles outside the pores are oxidized more easily than those inside the pores. Therefore, the micropores can prevent silver nanoparticles from being oxidized resulting in a substrate that can be used many times due to its long-term effectiveness.

The hot spots, which are the gaps between two silver nanoparticles that are dispersed on the three-dimensional porous membrane, make it possible for biomedical application. The special property of the substrate is investigated in a biomedical application. The pores in the membrane can trap Staphylococcus aureus (S. aureus) cells, and the Raman signal becomes stronger as more $S$. aureus cells are trapped.

\section{Conclusions}

In summary, we proposed a simple and low-cost method for fabricating a porous membrane decorated with silver nanoparticles, which is applied to a biomedical application. Two functionalities of the pores in the membrane are investigated, including (i) preventing the oxidation of silver nanoparticles and (ii) trapping bacteria to enhance the Raman signal.

\section{Disclosure}

Li Yuan and Tian Xu are co-first authors.

\section{Conflict of Interests}

The authors declare that there is no conflict of interests regarding the publication of this paper.

\section{Acknowledgments}

This work is supported by Ministry of Science and Technology of the People's Republic of China (no. 2011YQ03013403), National Natural Science Foundation of China (no. 61371057 and no. 11204143), Natural Science Foundation of the Jiangsu Higher Education Institutions of China (no. 13KJB140014), Open Fund of Key Subject of Physics, Zhejiang Province (no. xkzwl1522), and Jiangsu Students Project for Innovation Training (no. 201510304004Z). The authors would like to thank Xinxiang Ma, Jialei Miao, and Weijie Zhang for their contributions in the paper.

\section{References}

[1] G.-B. Jung, J.-H. Kim, J. S. Burm, and H.-K. Park, "Fabrication of chitosan-silver nanoparticle hybrid 3D porous structure as a SERS substrate for biomedical applications," Applied Surface Science, vol. 273, pp. 179-183, 2013.

[2] A. Barhoumi and N. J. Halas, "Label-free detection of DNA hybridization using surface enhanced Raman spectroscopy," Journal of the American Chemical Society, vol. 132, no. 37, pp. 12792-12793, 2010.

[3] G. Braun, J. L. Seung, M. Dante, T.-Q. Nguyen, M. Moskovits, and N. Reich, "Surface-enhanced raman spectroscopy for DNA detection by nanoparticle assembly onto smooth metal films," Journal of the American Chemical Society, vol. 129, no. 20, pp. 6378-6379, 2007.

[4] X. X. Han, B. Zhao, and Y. Ozaki, "Surface-enhanced Raman scattering for protein detection," Analytical and Bioanalytical Chemistry, vol. 394, no. 7, pp. 1719-1727, 2009.

[5] X. Wang, X. Qian, J. J. Beitler et al., "Detection of circulating tumor cells in human peripheral blood using surface-enhanced raman scattering nanoparticles," Cancer Research, vol. 71, no. 5, pp. 1526-1532, 2011.

[6] T.-Y. Liu, K.-T. Tsai, H.-H. Wang et al., "Functionalized arrays of Raman-enhancing nanoparticles for capture and culture-free analysis of bacteria in human blood," Nature Communications, vol. 2, article 538, 2011.

[7] M. Kahraman, M. M. Yazici, F. Şahin, and M. Çulha, "Convective assembly of bacteria for surface-enhanced Raman scattering," Langmuir, vol. 24, no. 3, pp. 894-901, 2008.

[8] B. D. Moore, L. Stevenson, A. Watt et al., "Rapid and ultrasensitive determination of enzyme activities using surfaceenhanced resonance Raman scattering," Nature Biotechnology, vol. 22, no. 9, pp. 1133-1138, 2004.

[9] A. J. Haes, S. Zou, G. C. Schatz, and R. P. Van Duyne, "Nanoscale optical biosensor: short range distance dependence of the localized surface plasmon resonance of noble metal nanoparticles," The Journal of Physical Chemistry B, vol. 108, no. 22, pp. 6961-6968, 2004.

[10] C. L. Haynes and R. P. Van Duyne, "Plasmon-sampled surfaceenhanced Raman excitation spectroscopy," The Journal of Physical Chemistry B, vol. 107, no. 30, pp. 7426-7433, 2003.

[11] T. R. Jensen, G. C. Schatz, and R. P. Van Duyne, "Nanosphere lithography: Surface plasmon resonance spectrum of a periodic array of silver nanoparticles by ultraviolet-visible extinction spectroscopy and electrodynamic modeling," Journal of Physical Chemistry B, vol. 103, no. 13, pp. 2394-2401, 1999.

[12] G. L. Liu and L. P. Lee, "Nanowell surface enhanced Raman scattering arrays fabricated by soft-lithography for label-free biomolecular detections in integrated microfluidics," Applied Physics Letters, vol. 87, no. 7, Article ID 074101, 2005.

[13] J. Wang, L. Huang, L. Zhai et al., "Hot spots engineering in hierarchical silver nanocap array for surface-enhanced Raman scattering," Applied Surface Science, vol. 261, pp. 605-609, 2012. 

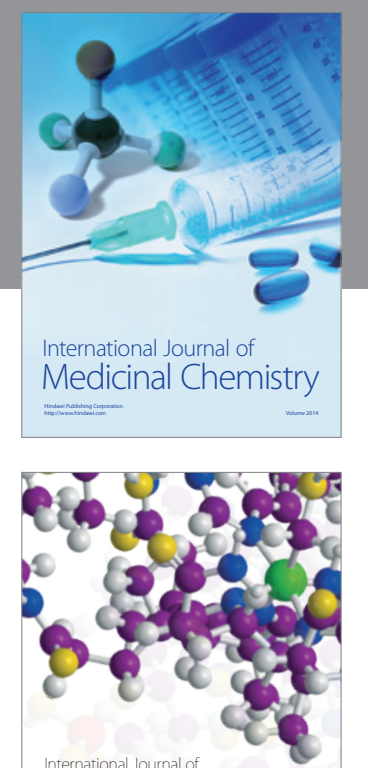

\section{Carbohydrate} Chemistry

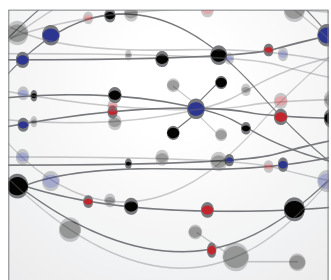

The Scientific World Journal
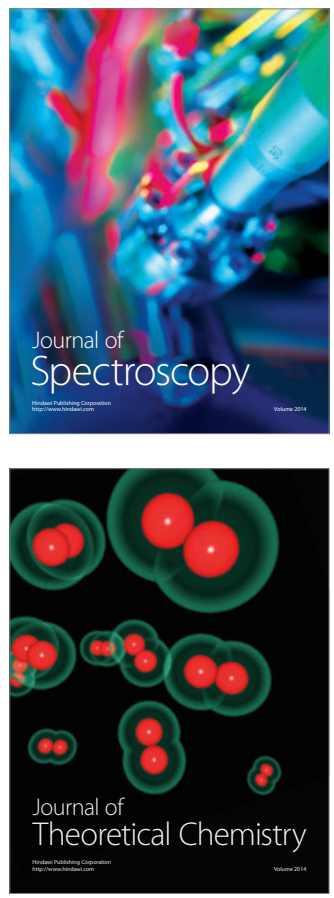
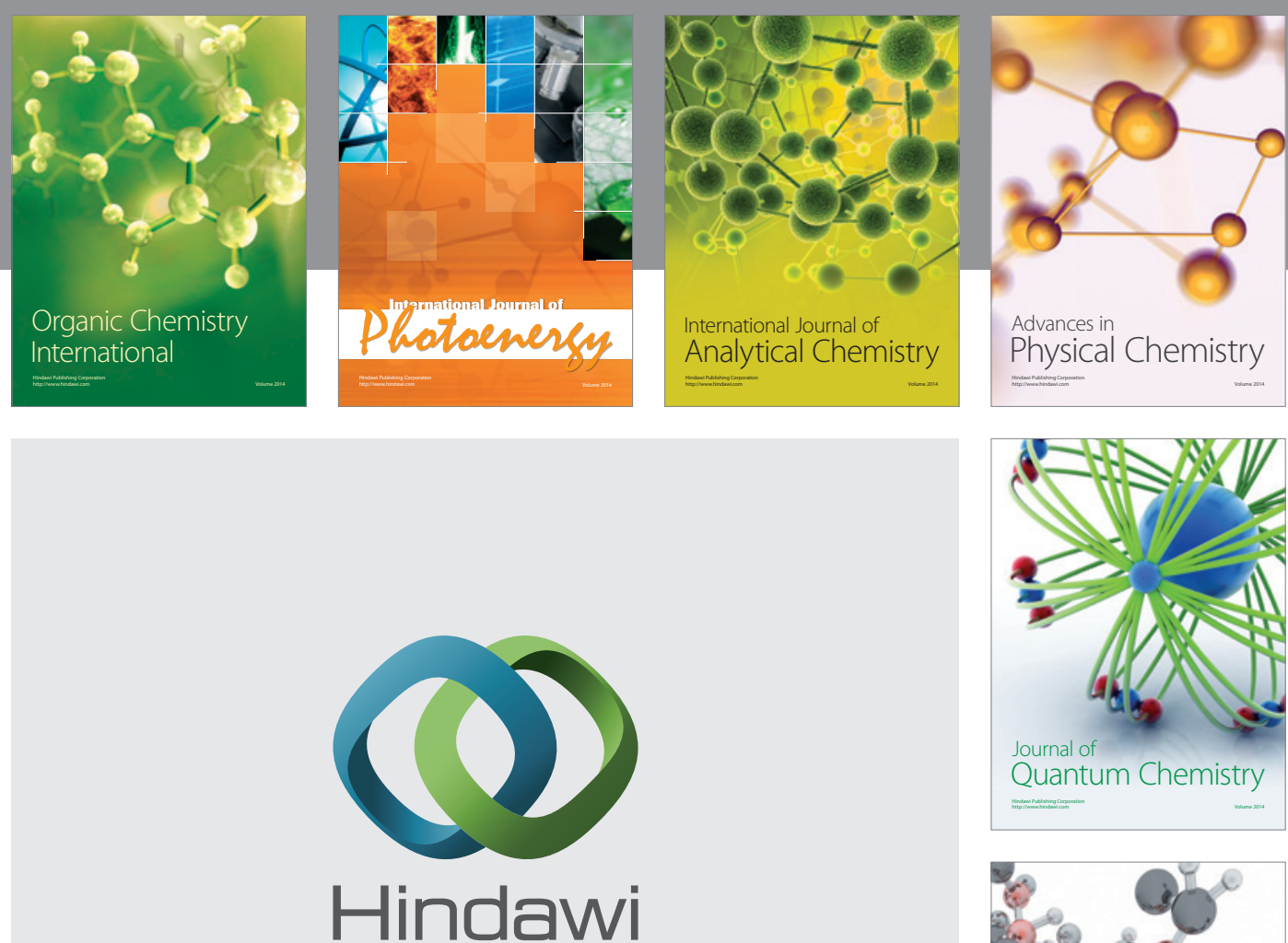

Submit your manuscripts at

http://www.hindawi.com

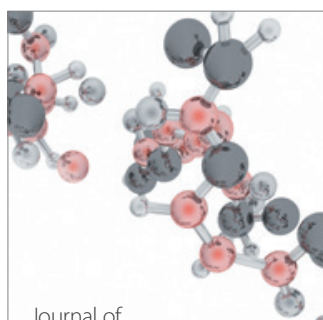

Analytical Methods

in Chemistry

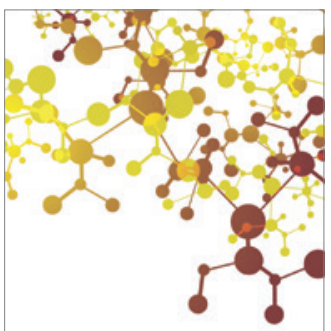

Journal of

Applied Chemistry

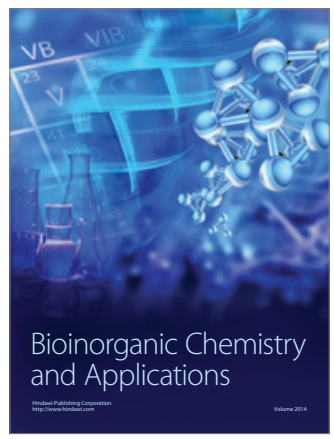

Inorganic Chemistry
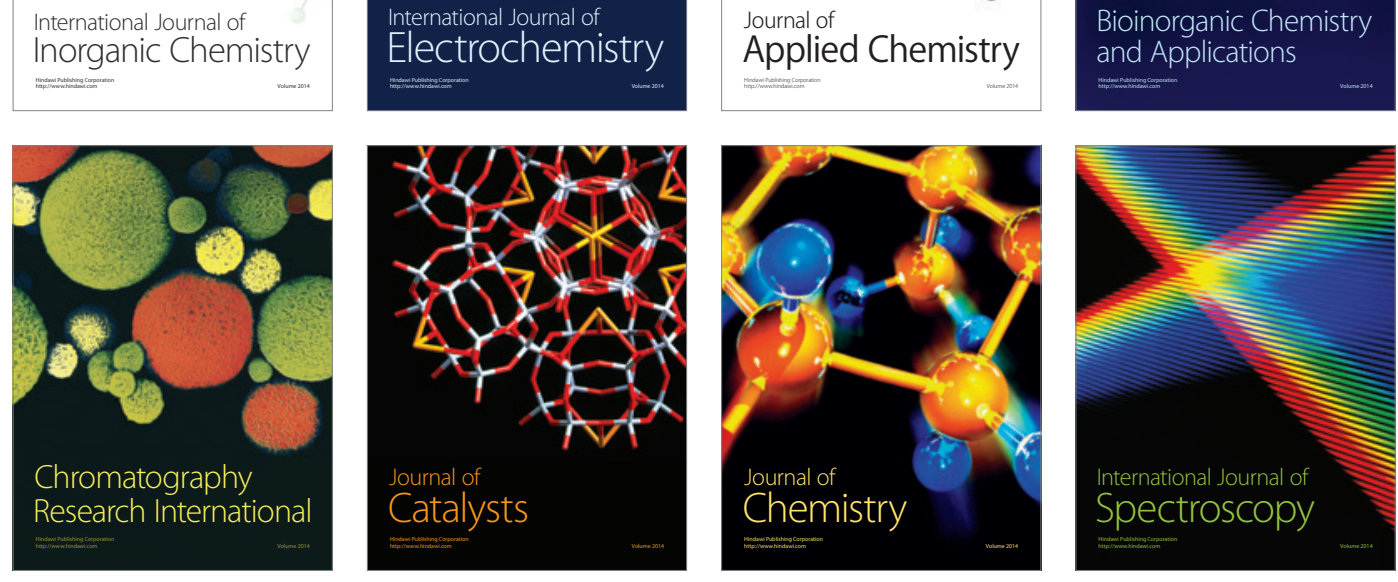